\title{
Revisión Sistemática del Estudio de las Motivaciones para la Parentalidad
}

\section{Revisão Sistemática do Estudo das Motivações para a Parentalidade}

\section{Systematic Review of Motivation to Parenthood Studies}

\section{Giuliana Vásquez Varas*}

Pontifícia Universidade Católica do Rio de Janeiro - PUC-Rio, Rio de Janeiro, Rio de Janeiro, Brasil

\section{J uliane Callegaro Borsa**}

Pontifícia Universidade Católica do Rio de Janeiro - PUC-Rio, Rio de Janeiro, Rio de Janeiro, Brasil

\begin{abstract}
RESUMEN
La presente revisión sistemática busca identificar, evaluar y sintetizar los estudios empíricos sobre Motivaciones para la Parentalidad publicados en ocho bases de datos electrónicas previamente escogidas. Producto de la combinación de descriptores en inglés, portugués y español se hallaron 1345 artículos que fueron evaluados a través de criterios de exclusión. La muestra final estuvo constituida por 49 artículos que fueron analizados en nueve categorías divididas entre aspectos generales y resultados de las investigaciones. Los resultados muestran un interés creciente en el estudio de las MP desde diferentes teorías pero se constató la carencia de estudios sobre esa temática en el contexto latinoamericano. El modelo TDIB de Warren Miller presentó una mayor aceptación para la instigación y medición del constructo. Se encontraron variables personales, culturales y contextuales relacionadas al estudio de las motivaciones para a parentalidad. Finalmente se describe diferentes limitaciones metodólogas que fueron mencionadas por los investigadores.
\end{abstract}

Palabras clave: fertilidad, motivación, hijos, parentalidad.

\section{RESUMO}

Esta pesquisa teve como objetivo identificar, avaliar e sintetizar os estudos empíricos sobre Motivação para a parentalidade (MP), publicados em oito bases de dados eletrônicas escolhidas. Da combinação de diversos descritores em inglês, português e espanhol, encontrou-se 1345 publicações internacionais. Após a submissão dos artigos ao refinamento inicial e teste de relevância, restaram 49 estudos que constituíram a amostra final desta pesquisa. Identificaram-se nove categorias analíticas divididas entre aspectos gerais e resultados dos estudos. Os resultados mostram um interesse crescente no estudo das MP desde diferentes teorias, embora constatou-se a carência de estudos no contexto latino-americano. O modelo TDIB de Warren Miller apresentou uma maior aceitação no estudo e 
mensuração do construto. Variáveis pessoais, culturais e contextuais relacionadas ao estudo das motivações para a parentalidade foram encontradas. Por fim, diferentes limitações metodológicas mencionadas pelos pesquisadores são descritas.

Palavras-chave: fertilidade, motivação, parentalidade.

\begin{abstract}
The current study aimed to identify empirical studies on Motivation to Parenthood (MP) through a systematic search in eight electronic databases. The combination of several descriptors in English, Portuguese and Spanish resulted in 1345 international publications. After initial refining and analysis, 49 studies met specifics inclusion criteria and remained to constitute the final sample of this search. Nine analytical categories were identified, divided between general aspects and studies results. The results indicate a growing interest and diverse theoretical approach in the study of MP. Nevertheless, it was verified a notable gap in this research field in Latin America context. The TDIB framework of Warren Miller to understand and measure MP was found to have great acceptance among scholars. Personal, cultural and contextual variables related to the study of motivation to parenthood were identified. Finally, methodological limitations presented by researchers are described.
\end{abstract}

Keywords: fertility, motivation, children, parenthood.

Latasa de fertilidad ha disminuido en la mayoría de países occidentales desde la segunda mitad del siglo XX. El número de hijos por mujer paso de 5 en el año 1960 a 2,5 en el 2015 (Banco Mundial, 2018). Estos cambios fueron producto de una serie de factores sociales y tecnológicos como la influencia de movimientos feministas y democráticos, así como el desarrollo y utilización de métodos anticonceptivos que modificaron el comportamiento reproductivo, antes considerado producto del azar para ahora ser ponderado como una elección consciente del sujeto (Miller, 1994). Actualmente, las personas pueden, con mayor grado de control, decidir si quieren tener hijos, así como el número y tiempo para tenerlos (Liefbroer, 2005), evidenciándose una reducción en la tasa de fecundidad e incremento en el número de mujeres que deciden no tener hijos (Leeners et al., 2009). Más, a pesar de todas esas trasformaciones, la parentalidad sigue siendo un objetivo central en la mayoría de las sociedades.

El término motivación ha sido largamente utilizado en compendios de psicología, en diferentes contextos y con diferentes significados. Del mismo modo, su conceptualización es abordada de maneras muy diferentes $y$, muchas veces, contradictorias (Todorov \& Borges, 2005). Segundo Miller, Severy y Pasta (2004), la motivación es considerada como una fuerza psicológica que impulsa a los individuos en dirección a un objetivo y fue definida como a disposición de sentir y comportarse de determinada forma, satisfaciendo una necesidad o alcanzando una meta do organismo. Por otro lado, el termino parentalidad, viene siendo utilizado para decir del estado o condición 
de padre o de madre, pudiendo ser explicado como el conjunto de las funciones y actividades desarrolladas por un progenitor o cuidador, con vistas al sano y pleno desarrollo del niño a su cargo (Ribeiro, Gomes, \& Nunes, 2015).

Las motivaciones para la parentalidad (MP) son consideradas como rasgos psicológicos o disposiciones latentes para percibir de forma favorable o desfavorable diferentes aspectos de la parentalidad que a la larga general el deseo de tener o no tener hijos (Gonçalves, 2016; Domingues, 2016). Se evidencian diferentes intentos de conceptualizar y explicar esa fuerza motriz por detrás da decisión de tener hijos, la mayoría producto do enfoque psicológico, social o económico predominante en la época y siempre intentando responder a una demanda socio histórica determinada (Matias \& Fontaine, 2013). Su estudio inició en el contexto de superpoblación de años sesenta con el objetivo de determinar el valor atribuido a los hijos en busca de alternativas a esos valores para reducir la intención de procrear (Gerson, 1983). Posteriormente, los cambios históricosociales generaron un declive en el índice de natalidad y transformaciones en el estudio de las MP; iniciando el análisis de la influencia de variables contextuales y psicológicas y el estudio del comportamiento reproductivo en individuos de diferentes contextos y condiciones (Beckman, 1987).

Teniendo en cuenta las características de los padrones de fecundidad, así como las condiciones históricas y sociales contemporáneas, el estudio de las MP se torna importante para la comprensión de la toma de decisiones reproductivas (Guedes, Pereira, Pires, Carvalho, \& Canavarro, 2013). En base a eso, el presente estudio tiene como objetivo describir como el estudio de las MP viene retratándose en el escenario internacional y cuáles son las variables e instrumentos que han sido considerados en ese proceso con la finalidad de presentar una contribución al área apuntando a los campos pobremente estudiados, así como aquellos que vienen siendo constantemente abordados.

\section{Método}

Se realizó una Revisión Sistemática de Literatura con la intención de maximizar el potencial de búsqueda de la temática escogida, encontrar y organizar la mayor cantidad de resultados y evaluar metódicamente los estudios obtenidos (Costa \& Zoltowski, 2014). Las siguientes etapas fueron recorridas:

Definición del objetivo: Realizar una Revisión Sistemática para describir el estudio de las MP en el escenario internacional e identificar cuáles son las variables e instrumentos que han sido considerados en ese proceso. 
Localización y selección de los estudios: Los descriptores fueron definidos a partir de la consulta de palabras-clave en terminología de psicología (BVS Psicología Brasil) y terminología en salud (Descriptores en Ciencias de la Salud - DeCS). Se utilizó los siguientes descriptores y operadores booleanos: (1) (Motivação OR motivación OR motivation) AND (2) (Parentalidade OR parentalidad OR parenting OR parenthood) OR (maternidade OR maternidad OR motherhood) OR (paternidade OR paternidad OR fatherhood) OR (childbearing).

La búsqueda de los artículos fue realizada en Agosto del 2018, en ocho bases de datos: (1) PsylNFO, (2) PePSIC, (3) PubMed, (4) ERIC,(5) JSTOR, (6) Portal de Periódicos CAPES, (7) LILACS y (8) SciELO. Se realizó "sin restricciones de fecha", con el objetivo de comprender la historicidad de los estudios. Se optó por realizar la búsqueda en las siguientes configuraciones generales: "asunto", "título" y "palabras-clave". Según las opciones disponibles en cada base de datos, se seleccionó los siguientes filtros para el cumplimiento de los criterios de inclusión: (1) Tipo de material: "artículo"; (2) idiomas: "inglés", "español" y "portugués" y (3) edad de los grupos: "participantes adultos". Los descriptores (parenthing or parenthood or parentalidade or parentalidad) y (motivation or motivação or motivación) recuperaron 672 artículos; (fatherhood or paternidade or paternidad) y (motivation or motivação or motivación) recuperaron 170, (motherhood or maternidade or maternidad) y (motivation or motivação or motivación) recuperaron 382 y childbearing y motivation recuperaron 121; dando en total 1.345 artículos.

Análisis crítico de los estudios: 1) Refinamiento inicial, donde se excluyeron artículos duplicados, incompletos e indisponibles. 2) Verificación de relevancia, donde se analizaron los resúmenes y se eliminaron los estudios conforme los siguientes criterios de exclusión: (a) análisis del lineamento de estudio (excluyéndose estudios teóricos, revisiones sistemáticas y meta análisis) y (b) análisis del objetivo y tema investigado (excluyéndose estudios que no tuvieron de tema principal las MP). La muestra final constó de 49 artículos que fueron leídos integralmente (Figura 1). 


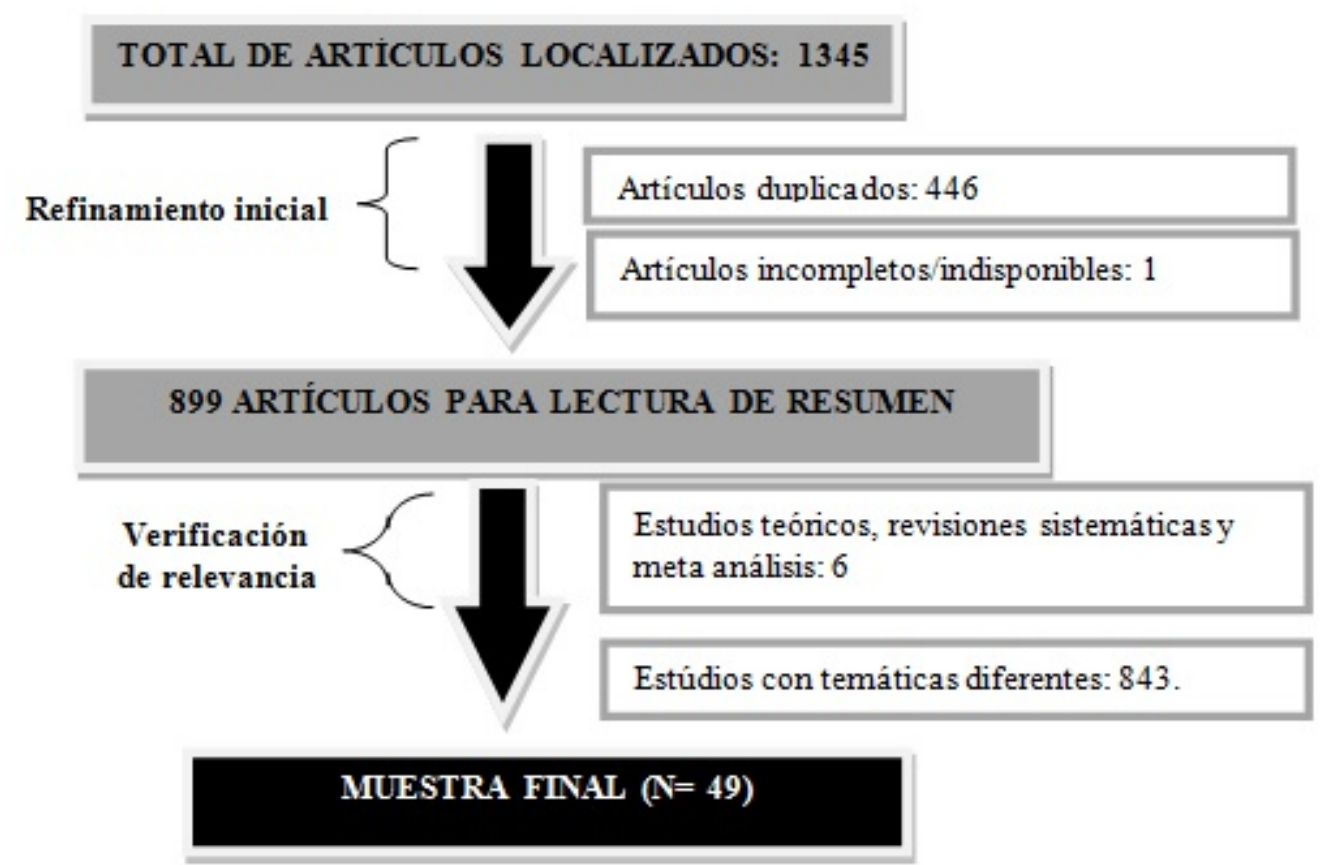

Figura 1. Flujograma.

\section{Resultados y Discusión}

Los 49 estudios recuperados fueron analizados en base a las siguientes categorías: (1) Aspectos generales de los estudios: a) año de publicación, b) contexto geográfico, c) delineamiento, d) características de la muestra, e) referencial teórico e g) instrumentos usados en la colecta de datos; (2) Resultados de los estudios: a) variables relacionadas al estudio de MP, b) principales resultados obtenidos y c) limitaciones/recomendaciones.

\section{Aspectos generales de los estudios}

Año de publicación: La investigaciónmásantiguaencontrada fue publicada en Estados Unidos de América (EUA) en el año 1965 y tuvo como objetivo el estudio de las MP y las actitudes hacia el matrimonio y educación de los hijos (Rabin, 1965). Desde 1980 hasta 2018, la producción científicase distribuyó de la siguiente manera: 1956-1989 $(n=9), \quad 1990-1999 \quad(n=10), \quad 2000-2010 \quad(n=13)$ y 2011-2018 $(n=17)$. Factores demográficos como la superpoblación en la década de 60 generó interés enel estudio de las MP (Gerson, 1983) y en la década de los 70, con el nacimiento de los movimientos feministas y democráticos, así como con los avances médico-tecnológicos relacionados a los métodos anticonceptivos; el panorama social cambió y con él, elestudio de las MP (Matías \& Fontaine, 2013). Pese 
al incipiente interés por estudiar las MP en los 60 y 70, la producción científica encontrada ha sido reducida. Este dato ya ha sido mencionado por otros autores como Brenning, Soenens, \& Vansteenkiste (2015) que manifestaron en sus escritos su preocupación por la escasez de estudios sobre MP y la necesidad de un análisis más profundo de este fenómeno.

Contexto geográfico del estudio: Se encontró quepredominan las investigaciones realizadas en EUA $(n=24,50 \%)$, país considerado como el más activo en producción científica nivel mundial y productor de la mayoría de estudios en el área de Humanidades, Ciencias Sociales, Ciencias Médicas y de la Salud (Kamalski et al., 2015). Retirando las publicaciones en los EUA, la producción encontrada se presentó de la siguiente manera: Europa: 10; Asia: 6; África: 3 y Oceanía: 2. También se encontraron cuatro estudios transculturales realizados en: 1) Camerún, Costa Rica, Alemania y China (Chasiotis, Bender, \& Hofer, 2014); 2) Inglaterra y Grecia (Cassidy \& Sintrovani, 2008); 3) Canadá, EUA y Francia (Jungert et al., 2014) y 4) EUA y Canadá (Stanford \& Porucznik, 2017). No se encontraron estudios sobre MP en países Latinoamericanos.

Delineamiento de los estudios: Predominaron las investigaciones de naturaleza cuantitativa $(n=30 ; 61 \%)$, de las cuales 23 fueron transversales, cinco longitudinales y dos análisis de datos secundarios. En los criterios de inclusión de los estudios, los investigadores delimitaron si las muestras estarían conformadas por individuos con o sin hijos. Al análisis realizado con grupos de participantes sin hijos lo denominaron: "análisis prospectivos de las MP" y a aquellos realizados ensujetos con hijos: "análisis retrospectivos". Se encontró 30 estudios (61,2\%) que realizaron análisis prospectivo, once $(22,4 \%)$ análisis retrospectivo y ocho (16,3\%) utilizaron ambos.

Características de las muestras: Treinta estudios (61,2\%) consideraron únicamente el sexo de los participantes o la presencia/ausencia de hijos como criterio de inclusión o exclusión y $19(38,8 \%)$ investigaron las MP en sujetos con otros requisitos previos como por ejemplo: infertilidad, orientación sexual, presencia de alguna enfermedad, etc. Se observa quela mayor parte de losestudiosfueron realizados enparejas sin hijos $(n=29 ; 50,2 \%)$ eincluyeron ambos sexos $(n=25 ; 51 \%)$. Influenciadas por las normas culturales de fertilidad, no solo el estudio de las MP variaroncon el tiempo sino también las variables de estudio relacionadas y las características de los sujetos pertenecientes a las muestras (Beckman, 1987). Un ejemplo de eso es el predominio de estudios que consideraron ambos sexos y la presencia, aunque reducida, de estudios con muestras exclusivamente masculinas $(n=2 ; 4 \%)$ que muestran mayor interés en la inclusión del hombre en el ámbito familiar y la consideración de ambos sexos en las decisiones 
reproductivas. Otros estudios producto de esos cambios sociohistóricos fueron los realizados con sujetos homosexuales, mujeres entratamiento de fertilización in vitro y parejas que ejercen la parentalidad tardía (Goldberg, Downing, \& Moyer, 2012).

Referencial teórico: Treinta y tres artículos (67.3\%) utilizaron modelos teóricos de MP que consideran estas como una dicotomía de aspectos positivos y negativos de tener hijos. Esta forma de entender la MP apareció en la década de los ochenta y tuvieron como base la teoría de Intercambio Social de Homans (Beckman, 1987) en la cual, las mudanzas en las interacciones humanas se originaban del análisis entre los costos y beneficios ofrecidos (Al-Fadhliy \& Smith, 1996; Beyeza-Kashesya et al., 2010).

Bajo una perspectiva cronológica, los primeros estudios sobre el valor de los hijos fueron los de Hoffman y Hoffman en 1973 que delimitaron nueve categorías distribuidas entre recompensas sociales, psicológicas/emocionales y económicas. Diez años después Fawcett realizó una categorización de costos económicos, laborales, psicológicos y físicos relacionados a los hijos (Matias \& Fontaine, 2013). Estos estudios fueron desarrollados en un contexto social diferente al actual como respuesta al crecimiento exacerbado de la población (Gerson, 1983).

Se encontró que catorce estudios usaron el modelo Motivos-DeseosIntenciones-Comportamientos (TDIB) de Miller (1995) el cual contextualiza las MP dentro de un enfoque integrativo de aspectos biológicos, sociales y comportamentales. En este enfoque las disposiciones motivacionales tienen base biológica, son modeladas por experiencias durante la infancia, y convertidas, bajo determinadas circunstancias, en deseos e intenciones de fertilidad conformando la base de dos comportamientos instrumentales: proceptivos e contraceptivos (Miller, Millstein, \& Pasta 2008). Este modelo ha sido testado empíricamente por el autor (Miller, 1994, 1995; Miller et al. 2004; Miller, Trent, \& Chung, 2014) y por otros investigadores como Pezeshki, Zeigham y Miller (2005), con resultados favorables que corroboran a relación entre los componentes del modelo.

También se encontró que ocho estudios consideraron a las MP como una fuerza psicológica general que impulsa al individuo a un objetivo, sin clasificarla en positiva-negativa. Dos de ellos, evaluaron la intensidad de la disposición para tener hijos (Boehne, 2010; Gerson, 1983); otro investigó la influencia de variables psicológicas, económicas, sociodemográficas así como la interacción con la pareja (Beckman, Aizenberg, Forsythe, \& Day, 1983); uno realizo un análisis de las MP relacionándolas con la teoría del miedo y la necesidad de transcendencia (Hoppe, Fritsche, \& Koranyi, 2016) y otro utilizo el modelo de interacción simbólica que refiere que una persona se 
define y elige formas de actuar basándose en las expectativas sociales impuestas (Wesley, 2003).

Otros enfoques utilizados en el estudio de las MP fueron: la teoría de la Autodeterminación de Deciy Ryan (Brenning et al., 2015; Dor \& Cohen-Fridel, 2010; Jungert et al., 2014); la teoría psicodinámica (Rabin, 1965); la psicología evolutiva del desarrollo (Chasiotis et al., 2014) y la teoría deconstruccionismo social (Goldberg et al., 2012).

Técnicas e instrumentos utilizados para evaluar las MP: La técnica más usada fue la escala de auto relato $(n=33 ; 67,3 \%)$, regularmente utilizada debido a que es rápida, económica y accesible (Cozby, 2002). Las investigaciones cualitativas utilizaron entrevistas $(n=12 ; 24,5 \%)$, ítemsaislados de escalas estructuradas $(n=1 ; 2 \%)$ (Al-Fadhli \& Smith, 1996), frases incompletas $(n=1 ; 2 \%)$ (Rabin, 1965) y actividades lúdicas ( $n=1 ; 2 \%)$ (Coney \& Mackey, 1998). Como se muestra la Tabla 1 , la escala más usada fue Miller's Chilbearing Questionnaire (CBQ) creado por el investigador norteamerico Warren Miller cuya teoría fue descrita anteriormente. Este instrumento fue utilizado en once estudios realizados entre 1992 y 2018 en EUA, I rán y Polonia. Otras escalas utilizadas para medir las MP fueron Index of Parenthood Motivation (Gerson, 1980), usada encinco estudios entre 1980 y 2003 y la Parenthood Motivation List (van Balen, 2004) utilizada en cuatro estudios entre 1998 y 2008.

Tabla 1

Características de Los Cuestionarios y Escalas Utilizadas para el Estudio de las MP

\begin{tabular}{|c|c|c|c|}
\hline Nombre & Autor & $\begin{array}{l}\mathrm{N}^{\circ} \text { de } \\
\text { estudios }\end{array}$ & País de estudio \\
\hline $\begin{array}{l}\text { Miller's Childbering } \\
\text { Ouestionnaire. }\end{array}$ & Warren B. Miller & 11 & $\begin{array}{l}\text { EUA } \\
\text { Irán }\end{array}$ \\
\hline $\begin{array}{l}\text { Index of Parenthood } \\
\text { Motivation. }\end{array}$ & Mary-Joan Gerson & 5 & EUA \\
\hline $\begin{array}{l}\text { The Parenthood Motivation } \\
\text { List. }\end{array}$ & $\begin{array}{l}\text { Van Balen y } \\
\text { Trimbos-Kemper }\end{array}$ & 4 & $\begin{array}{l}\text { Holanda, África del } \\
\text { Sur, Bélgica }\end{array}$ \\
\hline $\begin{array}{l}\text { Self Regulation Questionnaire } \\
\text { (Adaptación). }\end{array}$ & Ryan y Conne & 2 & $\begin{array}{c}\text { Israel, } \\
\text { Bélgica, } \\
\text { Transcultural }\end{array}$ \\
\hline $\begin{array}{l}\text { Parenthing Expectation } \\
\text { Questionnaire } \\
\text { Childbearing Motivation Scale }\end{array}$ & $\begin{array}{l}\text { O'Laughlin } \\
\text { Anderson } \\
\text { Guedes et al. }\end{array}$ & 2 & $\begin{array}{l}\text { EUA, } \\
\text { Israel } \\
\text { Portugal }\end{array}$ \\
\hline Operant Multimode Test & Kuhl y Scheffer & 1 & $\begin{array}{l}\text { Estudio } \\
\text { transcultural }\end{array}$ \\
\hline $\begin{array}{l}\text { Interest of Infants } \\
\text { Questionnaire }\end{array}$ & Charles et al. & 1 & China \\
\hline $\begin{array}{l}\text { Escala de motivos face a } \\
\text { parentalidade }\end{array}$ & Matias y Fontaine & 1 & Portugal \\
\hline Questionário de MP & Bell et al. & 1 & Escocia \\
\hline $\begin{array}{l}\text { Parenthood Motivation } \\
\text { Questionnaire }\end{array}$ & Seaver. & 1 & Israel \\
\hline Motives for Parenthood & Langdridge et al. & 1 & Inglaterra \\
\hline
\end{tabular}


Resultados de los estudios

Variables relacionadas al estudio de las MP: Las variables de estudio relacionadas a las MP fueron organizadas en las siguientes categorías: (1)experiencias en la infancia, (2) variables sociodemográficas de la muestra, (3) recursos y características personales de los participantes, (4) interacción actual con el contexto familiar y conyugaly (5) problemas de salud de los participantes. 
Tabla 2

Categorías y Subcategorías de las Variables

\begin{tabular}{|c|c|c|c|}
\hline Tipo & Variable & $\begin{array}{l}N^{\circ} \text { de } \\
\text { estudios }\end{array}$ & País \\
\hline \multirow{9}{*}{$\begin{array}{l}\text { Experiencias con } \\
\text { la familia de } \\
\text { origen }\end{array}$} & $\begin{array}{l}\text { Experiencias de cuidado en la } \\
\text { infancia }\end{array}$ & 6 & EUA \\
\hline & Felicidad en la vida familiar inicial. & 3 & EUA \\
\hline & $\begin{array}{l}\text { Status socioeconómico en la } \\
\text { infancia. }\end{array}$ & 1 & Transcultural \\
\hline & Religión & 7 & EUA \\
\hline & Etnicidad & 2 & EUA \\
\hline & Status socioeconómico & 5 & Transcultural \\
\hline & Número de hijos & 2 & EUA \\
\hline & Sexo & 1 & Transcultural \\
\hline & Número y orden de hermanos & 1 & Transcultural \\
\hline \multirow{11}{*}{$\begin{array}{l}\text { Categorías } \\
\text { sociodemográficas }\end{array}$} & Edad & 6 & EUA e Irán \\
\hline & Estado civil & 3 & EUA \\
\hline & Simpatía con políticas feministas & 2 & EUA \\
\hline & Nivel educativo & 2 & EUA e Irán \\
\hline & Trabajo actual & 1 & EUA \\
\hline & Edad cuando contrajo matrimonio & 1 & Irán \\
\hline & Orientación sexual & 2 & $\begin{array}{l}\text { Holanda y } \\
\text { EUA }\end{array}$ \\
\hline & Roles sexuales tradicionales & 7 & $\begin{array}{l}\text { EUA y } \\
\text { Holanda }\end{array}$ \\
\hline & Satisfacción de la vida & 2 & $\begin{array}{l}\text { EUA y } \\
\text { Bélgica }\end{array}$ \\
\hline & Apego & 1 & China \\
\hline & Autoestima y autoeficacia & 3 & EUA \\
\hline \multirow{13}{*}{$\begin{array}{l}\text { Recursos y } \\
\text { características } \\
\text { personales de los } \\
\text { participantes }\end{array}$} & Objetivos educativos & 1 & Holanda \\
\hline & Actitudes frente al casamiento & 1 & EUA \\
\hline & Personalidad & 3 & EUA \\
\hline & Estrategias de afrontamiento & 1 & Inglaterra \\
\hline & Angustia psicológica & 1 & Inglaterra \\
\hline & Creencias sobre fertilidad & 1 & EUA \\
\hline & Deseos de tener hijos & 4 & $\begin{array}{l}\text { EUA, Polonia } \\
\text { y China }\end{array}$ \\
\hline & Intenciones de tener hijos & 3 & $\begin{array}{l}\text { EUA, } \\
\text { Canadá, } \\
\text { China y } \\
\text { Polonia }\end{array}$ \\
\hline & Síntomas depresivos & 1 & Bélgica \\
\hline & $\begin{array}{l}\text { Comportamiento sexual relacionado } \\
\text { a la fertilidad }\end{array}$ & 2 & $\begin{array}{l}\text { EUA y } \\
\text { Canadá }\end{array}$ \\
\hline & Narcisismo & 1 & EUA \\
\hline & Influencia normativa percibida & 1 & EUA \\
\hline & Poder relativo & 1 & EUA \\
\hline \multirow{8}{*}{$\begin{array}{l}\text { Interacción actual } \\
\text { con el contexto } \\
\text { familiar y } \\
\text { conyugal }\end{array}$} & Apertura a la comunicación & 1 & EUA \\
\hline & Deseos de tener hijos de la pareja & 1 & EUA \\
\hline & Satisfacción marital & 2 & $\begin{array}{l}\text { Israel y } \\
\text { Bélgica }\end{array}$ \\
\hline & Coerción reproductiva & 1 & EUA \\
\hline & Apoyo social percibido & 1 & Inglaterra \\
\hline & HIV & 2 & EUA \\
\hline & Cáncer & 1 & Israel \\
\hline & Hipertensión & 1 & Alemania \\
\hline \multirow[t]{2}{*}{ Enfermedades } & Infecciones de transmisión sexual & 1 & $\begin{array}{c}\text { EUA } \\
\text { África del }\end{array}$ \\
\hline & Infertilidad & 4 & $\begin{array}{l}\text { Sul, Bélgica } \\
\text { y EUA }\end{array}$ \\
\hline
\end{tabular}


Como se puede observar en la tabla 1 , en la categoría 2 existe preponderancia de estudios que relacionan la MP con la edad y religión así mismo, en la categoría 3, la variable identificación con los roles de género fue la más investigada (Miller, 1992). La importancia de las experiencias en la niñez también fue un aspecto ampliamente estudiado, desde los estudios de Gerson en 1980 hasta la actualidad (Cheng, Zhang, Sun, Jia, \& Ta, 2015). Dato que se ajusta a la teoría actual de las MP que las describe como producto de la interacción entre una base biológica y experiencias en la infancia (Miller, Severy, \& Pasta, 2004).

También se observan estudios de las MP en sujetos que padecen enfermedades como: HIV, cáncer, hipertensión, infertilidad y infecciones de trasmisión sexual. La infertilidad con individuos en tratamiento de fertilización in vitro fue la más estudiada $(n=4)$, evidenciando que los avances médico-tecnológicos generaron una nueva forma de vivir y percibir la parentalidad cuyos efectos en la subjetividad aún están por determinarse.

Principales resultados obtenidos: a modo de organización serán descritos primero las principales MP encontradas para posteriormente pasar a los resultados de las principales variables demográficas, contextuales e individuales relacionadas a su estudio. Los estudios evidencian que las MP positivas predominantes en ambos sexos están relacionadas con las alegrías ocasionadas por el embarazo, nacimiento y cuidado de los hijos (Alexander, Trister, Sacko, Morgan, \& Arrington, 2018; Dyer, Mokoena, Maritz, \& van der Spuy, 2008; van Balen 2004). La afirmación de la identidad, crear legados personales, corregir historias familiares traumáticas y mejora de la relación conyugal fueron otras de las PM encontradas (Alexander et al., 2018; Braun, Hasson-Ohayon, Perry, Kaufman, \& Uziely, 2005; Dyer et al., 2008). En países no occidentales, como Uganda, las MP están influenciadas por aspectos relacionados al patriarcado y respetabilidad social (Beyeza-Kashesya et al., 2010). Braun et al. (2005) encontraron que la posibilidad de un hijo defectuoso, preocupación y responsabilidad que genera la crianza y consideraciones financieras fueron las MP negativas mayormente referidas.

Los variados resultados de las MP positivas y negativas deben ser entendidas como el resultado de diversas experiencias de vida de la persona así como por las exigencias sociales impuesta relacionadas a la maternidad y paternidad (Miller, 1994) por lo que es de extrema necesidad obtener datos ajustados a cada realidad poder entender la toma de decisiones reproductivas de cada cultura y realidad.

Diversas variables sociodemográficas fueron consideradas en el estudio sobre las MP, siendo la religión una de las más estudiadas. Se encontró evidencia empírica en la relación entre MP positiva y 
afiliación religiosa (Al-Fadhli \& Smith, 1996; Ghazanfarpour et al., 2018; Ngu, Hay, \& Menahem, 2015) y otros autores especificaron que la relación no se da con las MP sino con el deseo e intención de tener hijos (Beckman et al., 1983; Gerson, 1980). Definitivamente los estudios indican que la religión es una variable de influencia en la toma de decisiones reproductivas tanto en las motivaciones como en su posterior planificación debido a que está asociada a un sistema de valores fuertemente orientados a la familia (Miller \& Pasta, 1993). Enrelación a las diferencias entre las MP según la presencia o ausencia de hijos también se encontró evidencia empírica que lo sustenta. Beckman (1987) encontró que las parejas que tienen un hijo presentan menor motivación para tener otro y Stöbel-Richter, Beutel, Finck y Brähler (2005), que las parejas con hijos manifiestan mayor intención de procrear. Con esta variable también se evidencia influencia en la toma de decisiones reproductivas tanto en las motivaciones como en las intenciones de fertilidad, dato que debe ser tomado en cuenta a razón de que aunque deseos e intenciones elementos del esquema de comportamiento reproductivo, componen partes diferentes de este (Miller, 1995). Se evidencia necesidad de datos sobre las MP y las intenciones de las personas para tener un segundo o tercer hijo considerando que estas MP estarán influenciadas por experiencias previas de paternidad, así como por los deseos individuales y de la pareja (Miller \& Pasta, 1993).

Estudios encontraron que los varones percibieron menos costos asociados a un segundo hijo (Beckman, 1987) y que las mujeres manifestaron mayor intención de tener el primer hijo (Stöbel-Richter et al., 2005). Estando las mujeres sujetas a una fuerte presión social para convertirse en madres, se entiende la evidencia que describe una mayor manifestación de su intención de tener un primer hijo que los varones. Por otro lado, el hecho de la que las mujeres aún sean las principales responsables por el cuidado de los niños explica que los costos percibidos para tener un segundo hijo sean mayores que el de los varones (Stöbel-Richter et al., 2005).

Los resultados de las comparaciones de las MP de hombres y mujeres son variados. Algunos estudios mencionan que no hay diferencias entre motivación positiva entre varones y mujeres (Alexander et al., 2018), pero si en las negativas (Pezeshki et al., 2005), considerando la presencia de MP positiva en ambos sexos como reflejo de la valoración social otorgada a tener descendencia, en cuanto la mayor valoración de los aspectos negativos enlas mujeres refleja la mayor responsabilidad de cuidado de los hijos sobre el sexo femenino. Otros estudios describen diferencias entre varones y mujeres basadas enlas características de roles de género. Rabin (1965) mencionó que las MP del varón estaban basadas en la autorrealización, perpetuación y prueba de virilidad, en cuanto en la mujer cumplían funciones instintivas y predestinadas. Gerson (1980) refirió que las MP 
psicológicas eran mayormente mencionadas en las mujeres y las MP instrumentales en los varones y Miller (1992) encontró diferencias en relación a la búsqueda de afiliación en las MP positivas del hombre y de nutrición en la mujer. Es importante recordar que el análisis de los datos sobre diferencias entre varones y mujeres deben ser realizados considerando los diferentes roles sociales atribuidos a cada sexo.

Se encontró que el nivel educativo correlaciona negativamente con la motivación positiva en ambos sexos (Ghazanfarpour et al., 2018; Pezeshki et al., 2005). Estos datos contradicen lo descrito por Miller (1992) sobre que el nivel educativo disminuye la motivación en mujeres más no en varones. Esas discordancias pueden ser analizadas bajo la luz de los cambios sociales en relación al rol del varón en el cuidado de los hijos que origina una redistribución de prioridades en la planificación de la paternidad (Coney \& Mackey, 1998). Por otro lado, la educación expande la visión del individuo más allá de la familia y la comunidad de origen promoviendo el acceso a actividades diferentes a la parentalidad (Miller, 1992), originando individuos con altos niveles de educación que optan por posponen la paternidad o manifiestan negativa definitiva a la procreación (van Balen, 2004) ya que atribuyen menos valor a familias grandes y perciben mayores costos y menor beneficios en la parentalidad (Miller \& Pasta, 1993).

Miller (1992) relacionó la MP y la participación en el campo laboral, encontrando que las mujeres que tenían trabajo remunerado presentaban menor MP positiva y mayor MP negativa que sus parejas. Actualmente las mujeres responden a cambios en las condiciones socioeconómicas, así como a ideales sociales sobre el matrimonio y el parto mientras que los varones enfrentan un rol más activo y comprometido en la crianza de los hijos (Ngu et al., 2015) lo cual puede generar disminución en la valoración positivas de la maternidad en las mujeres y valoración de mayores costos y obligaciones en la paternidad en los varones.

La relación de las MP y la edad fue una de las variables más estudiadas en mujeres y los resultados fueron contradictorios. Algunos describen que las mujeres con mayor edad presentan MP, deseos e intenciones de fertilidad mayores a corto plazo (Beckman, 1987) al tener en cuenta su limitado periodo reproductivo y el aumento de la presión social (Colpin, De Munte, \& Mndemeulebroecke, 1998; Ngu et al., 2015). Otros afirman que a mayor edad menor motivación positiva (Miller, 1994) y menor deseo de tener hijos (Gerson, 1980). En relación a que existe una disminución de la MP y deseo de tener hijos con la edad, Miller y Pasta (1993) proponen dos argumentos explicativos: la consolidación del interés en proyectos y estilos de vida que la aleja de la idea de tener descendencia y los cambios biológico relacionados al 
envejecimiento que reducen el impulso y la energía que se requiere para criar un hijo.

Por otro lado, estudios realizados en mujeres jóvenes encontraron también resultados contradictorios; por un lado, Gerson (1980) halló que las mujeres más jóvenes consideraban menos atractiva la idea de tener hijos y estaban menos motivadas a superar los costos de la maternidad y por otro, van Balen (2004) encontró que ellas tenían más deseos por tener hijos que las mujeres con más edad. Las muestras masculinas también recibieron resultados diferentes. Algunos apuntaron que la influencia de la edad agravaba el incremento de las MP negativas en los varones (Beckman et al., 1983) y otros que ellos no demuestran diferencias relacionadas con esa variable (van Balen, 2004).

La edad es considerada como un factor importante en la reproducción humana, especialmente en las mujeres, tomando en cuenta la limitación temporal de su capacidad reproductiva y la presión social. La edad de inicio de la reproducción aumentó a medida que las mujeres participaron en otros campos, tornándose importante el estudio de esa variable en relación al valor cultural que se otorga a los hijos, el rol de la mujer en la sociedad y la inclusión del hombre en las actividades de crianza para así tener una comprensión contextualizada de esta variable en su relación con las MP (Miller et al., 2004).

Los estudios de las diferencias de la MP según la orientación sexual, fue resultado de los cambios sociales relacionados a las nuevas concepciones de familia. Se encontraron similitudes entre las MP de parejas homosexuales y heterosexuales propios de procesos de toma de decisiones en el curso de la vida, así como diferencias que reflejan preocupaciones formadas por su carácter de minoría sexual. El estudio de Bos, van Balen y van den Boom (2003) con parejas lésbicas apuntó que estas pasaban mayor cantidad de tempo reflexionando sobre sus MP y que la felicidad era el motivo principal para tener hijos y el estudio de Goldberg et al. (2012) con parejas gay que adoptaron, indicó que el deseo de enseñar tolerancia y de ofrecer un hogar eran propios de esas parejas.

Otra variable, producto de cambios sociohistóricos, es la asimilación de ideales feministas y las MP. Gerson encontró que las mujeres que se identificaban con movimientos feministas estaban menos interesadas en tener hijos, mas también que esas diferencias no se relacionaban con una valoración menos positiva de la maternidad. El interés en esta variable corresponde a una proliferación de los movimientos feministas en la década de 80 generando interés en encontrar relación con el comportamiento reproductivo (Gerson, 1984), pero desafortunadamente en la actualidad no se han desarrollado más estudios sobre el tema. 
Respecto a las experiencias con la familia de origen; Miller (1995) menciona que las vivencias en la niñez, como la calidad de las relaciones con los padres y transmisión de valores centrados en la familia son la base del desarrollo de disposiciones motivacionales positivas o negativas en relación a los hijos. Esas premisas ya habían sido exploradas en los estudios de Gerson (1980) al describir que recuerdos positivos de amor materno en la primera infancia explicaban el deseo por tener hijos. Gerson (1980) también realizo un estudio analizando la influencia del número de hermanos, encontrando que las mujeres que crecieron en familias numerosos (tres o más hermanos) expresaban mayor deseo de tener hijos que las mujeres de familias menores. Otro dato encontrado fue que el orden de nacimiento no estaba relacionado con las MP pero sí la calidad de la experiencia interactiva con los hermanos ya que constituye un aspecto fundamental enel contexto infantil por lo que vivencias positivas o negativas en esa relación explica una gran variedad de fenómenos en la edad adulta, como por ejemplo las MP (Chasiotis et al., 2014).

Otro constructo estudiado en su relación con las MP fue la identificación con los roles sexuales tradicionales (Beckman et al., 1983; Gerson, 1980; Leeners et al., 2009). En esos estudios se consideró esa variable como un rasgo de personalidad asociado a MP específicas. Otra propuesta de análisis de género fue que la parentalidad sirve como un medio para cumplir exigencias relacionadas a los roles masculino y femenino (Coney \& Mackey, 1998; Goldberg et al., 2012; Guedes et al., 2013) que acaba repercutiendo tanto en las intenciones de tener hijos como en la propia fertilidad (Miller \& Pasta, 1993).

En relación a la personalidad, Miller (1995) encontró que rasgos de nutrición en mujeres y de afiliación en varones preveían la motivación positiva para tener hijos, considerando que estos rasgos disponen positivamente a los individuos al cuidado de un otro. La autonomía fue la característica de personalidad que mejor predijo la motivación negativa al considerar a los niños como dependientes para su supervivencia. Gerson (1980) también sustentó que las MP varían según las características de personalidad más esa relación no fue profundamente analizada.

Otras variables asociadas positivamente a las MP fueron satisfacción positiva con la vida (Al-Fadhli\& Smith, 1996; Brenning et al., 2015; Wesley, 2003) y estrategias de afrontamiento (Cassidy \& Sintrovani, 2008). Las variables autoestima y autoeficacia fueron estudiadas en muestras femeninas con y sin el virus de inmunodeficiencia humana (VIH) (Wesley, 2003), encontrándose que ambas variables correlacionan positivamente con el deseo de tener hijos en ambos grupos. Esos datos difieren del estudio de Gerson (1980) en el cual las mujeres con baja autoeficacia tenían un mayor deseo por tener 
hijos ya que encaran la maternidad como una compensación de sus deficiencias al intentar reestablecer su autoestima a través del servicio.

La influencia del esquema motivacional de la pareja fue otra variable estudiada por medio del análisis de la influencia normativa, poder relativo, apertura a la comunicación (Beckman et al., 1983), las motivaciones percibidas por las parejas (Alexander et al., 2018) y satisfacción marital (Brenning et al., 2015). Es seguro decir que los humanos normalmente tienen la capacidad de percibir cada componente de la toma de decisiones reproductivas de sus consociados, incluidos los rasgos conscientes e inconscientes. Como discutieron Miller y Rodgers (2001), la capacidad de comunicar estados internos y la capacidad de interpretar esa comunicación son funciones críticas de los esquemas de vinculación que han evolucionado conjuntamente a lo largo del tiempo evolutivo para garantizar una precisión razonable. Es probable que uno cambie la propia estructura motivacional en respuesta a las percepciones de la estructura motivacional de la pareja íntima. Este proceso a menudo ocurre con el tiempo y refleja una aceptación del punto de vista del cónyuge, un resultado natural de la intimidad dentro de la díada (Miller et al., 2004).

Un número limitado de estudios se enfocó en comparar las MP en diferentes culturas. $Y$ los pocos encontrados mencionan como limitación el número reducido de participantes que dificultó la comparación y discusión posterior (Cassidity \& Sintrovani, 2008; Coney \& Mackey, 1998). Los estudios transculturales son necesarios para profundizar en la comprensión de temáticas con influencias contextuales variadas como es el caso de las MP que tienen como base las experiencias del sujeto y el contexto en el que se desenvuelve (Miller, 1994).

Se observa tres tipos de limitaciones enlos estudios encontrados: problemas con el análisis de datos, dificultades enla operacionalización de variables y dificultades relacionadas a las muestras. Sobre el análisis de datos se menciona el uso de un modelo inapropiado para encontrar las relaciones pretendidas y queel índice del tamaño del efecto, aunque respetable para pesquisas enciencias sociales, no era grande (Beckman, 1987). Las limitaciones basadas en las dificultades de operacionalización se dieron en variables como poder relativo e interacción de la pareja (Beckman et al., 1983). La limitación más mencionada fue la relacionada a la composición de las muestras (Guedes et al., 2013), representación desigual en género, estado civil, nivel de instrucción y número de hijos que dificultaba la generalización de resultados (Cheng et al. 2015, Dor \& Cohen-Fridel, 2010; Golberg et al., 2012), muestras no aleatorias que limitaban las inferencias extraídas de los estudios comparativos (Wesley, 2003) y tamaño pequeño que complicaba el 
encontrar diferencias significativas entre los grupos (Braun et al., 2005; Dyer et al., 2008; Colpin et al., 1998; Ngu et al., 2015). Menos mencionado fue el uso de instrumentos no validados (Cassidity \& Sintrovani, 2008) y el hecho de que el estudio haya sido desarrollado en personas que ya tienen hijos (Agustine et al., 2009; Colpin et al., 1998). Frente a esas limitaciones los autores recomendaron que los resultados sean interpretados con cautela, el uso futuro de estudios longitudinales, cuidado en la homogeneidad de las muestras y la participación de sujetos en diferentes etapas de su vida.

\section{Consideraciones Finales}

El estudio tuvo como objetivo realizar una revisión sistemática para identificar, evaluar y sintetizar los estudios empíricos sobre MP con la intención de contribuir en la construcción de conocimiento sobre el área, considerando la complejidad de los aspectos envueltos en la comprensión de esta temática. A partir de esta revisión, se enfatiza la importancia del estudio de las MP como parte del desarrollo humano con una base teórica que integre diversos elementos individuales y contextuales. Por otro lado, considerando que la comparación del funcionamiento psicológico en diferentes culturas posibilita probar los supuestos universales sobre el comportamiento humano y las relaciones sociales, resulta necesario establecer estrategias que permitan comparar los datos recolectados en diferentes culturas, respetando las peculiaridades de cada individuo e intentando atribuir cierto grado de universalidad a los resultados obtenidos (Allik \& Realo, 2004; Dasen \& Mishra, 2000).

Fue posible visualizar escases de estudios en Latinoamérica y pocas producciones a nivel mundial, por lo que hacemos énfasis en la necesidad de estudios sobre el tema. También se encontró instrumentos de evaluación de las MP, la mayoría de ellos basados teóricamente en la existencia de MPs positivas y negativas, así como resultados diversos sobre influencia de variables individuales y contextuales en el tiempo y en diferentes países. Se considera oportuno resaltar que una limitación de este trabajo es que los artículos fueron resultado de una búsqueda a partir de criterios ya establecidos, como la elección de bases de datos e descriptores; por lo que se recomienda nuevas búsquedas en otras bases de datos y nuevos descriptores en las futuras investigaciones, para una constante actualización del tema. 


\section{Referencias}

Alexander, K., Trister, K., Sacko, C., Morgan, A., \& Arrington, R. (2018). "Having child mean anti had a real life": Childbearing motivations and reproductive coercion among urban socioeconomically disadvantaged black young men. Journal of Adolescent Health, 62(2), 1-19. doi: 10.1016/j.jadohealth. 2017.11.037

Al-Fadhli, H. M., \& Smith, J. C. (1996). Assessing the impact of violence on motivation for parenthood. Journal of Negro Education, 65(4), 424-433. doi: 10.2307/2967145

Allik, J., \& Realo, A. (2004). Individualism-Collectivism and Social Capital. Journal of Cross-Cultural Psychology, 35(1), 29-49. doi: $10.1177 / 0022022103260381$

Banco Mundial. (2018). Taxa global de fecundidade. Recuperado de https: //datos. bancomundial.org/indicador/SP.DYN.TFRT.I N

Beckman, L. (1987). Changes in Motivation for parenthood among young married couples. Population and Environment, 9(2), 96110. doi: 10.1007/BF01258274

Beckman, L., Aizenberg, R., Forsythe, A., \& Day, T. (1983). A theorical analysis of antecedents of young couples fertility decisions. Demography, 20(4), 519-533. doi: 10.2307/2061117

Beyeza-Kashesya, J., Neema, S., Ekstrom, A. M., Kaharuza, F., Mirembe, F., \& Kulane, A. (2010). "Not a boy, not a child": A qualitative study on young people's views on childbearing in Uganda. African Journal of Reproductive Health, 14(1), 71-81. Recuperado de http://www.bioline.org.br/pdf?rh10006

Boehnke, M. (2010). To have or not to have: On fine distinctions in childbearing motivation among childless east and west german women. International Journal of Sociology of the Family, 36(1), $1-23$. Recuperado https://www.jstor.org/stable/23070776?seq=1\#page_scan_tab contents

Bos, $\bar{H}$. M., Van Balen, F., \& Van Den Boom, D. (2003). Planned lesbian families: their desire and motivation to have children. Human Reproduction, 18(10), 2216-2224. doi: 10.1093/humrep/deg427

Braun, M., Hasson-Ohayon, I., Perry, S., Kaufman, B., \& Uziely, B. (2005). Motivation for giving birth after breast cancer. PsychoOncology, 14(4), 282-296. doi: 10.1002/pon.844

Brenning, K., Soenens, B., \& Vansteenkiste, M. (2015). What's your motivation to be pregnant? relations between motives for parenthood and women's Prenatal Functioning. Journal of Family Psychology, 29(5), 755-765. doi: 10.1037/fam0000110

Cassidy, T., \& Sintrovani, P. (2008). Motives for parenthood, psychosocial factors and health in women undergoing IVF. 
Journal of Reproductive Psychology, 26(1), 4-17. doi: 10.1080/02646830701691392

Chasiotis, A., Bender, M., \& Hofer, J. (2014). Childhood context explains cultural variance in implicit parenting motivation: results from two studies with six samples from Cameroon, Costa Rica, Germany, and China. Evolutionary Psychology, 12(2), 295-317. doi: 10.1177/147470491401200203

Cheng, G., Zhang, D., Sun, Y., Jia, Y., \& Ta, N. (2015). Childless adults with higher secure attachment state have stronger parenting motivation. Personality and Individual Differences, 87, 39-44. doi: 10.1016/j. paid.2015.07.004

Colpin, H., De Munte, A., \& Mndemeulebroecke, L. (1998). Parenthood motives in IVF-mothers. Journal of Psychosomatic Obstetrics y Gynecology, 19(1), 19-27. doi: 10.3109/01674829809044217

Coney, N. S., \& Mackey, W. (1998). Motivations toward fathering: Two minority profiles within the majority's context. The Journal of Men's Studies, 6(2), 169-188. doi: $10.1177 / 106082659800600204$

Costa, A. B., \& Zoltowski, A. P. C. (2014). Como escrever um artigo de revisão sistemática. In S. H. Koller, M. C. P. Paula Couto, \& J. V. Hohendorff (Orgs.), Manual de Produção Científica (pp. 5570). Porto Alegre, Brasil: Penso.

Cozby, P. (2002). Métodos de pesquisa em ciências do comportamento. São Paulo, Brasil: Atlas.

Dasen, P. R., \& Mishra, R. C. (2000). Cross-cultural views on human development in the third millennium. International Journal of Behavioral Development, 24(4), 428-434. doi: 10.1080/016502500750037973

Domingues, C. F. (2016). Motivações para a Parentalidade em Futuros Pais Adotivos e Futuros Pais Biológicos. (Tese de Doutorado). Instituto Superior Miguel Torga, Coimbra, Portugal.

Dor, A., \& Cohen-Fridel, S. (2010). Perceived parenthood: Crosscultural differences between jewish and arab emerging adults. The Journal of Adult Development, 17(1), 12-19. doi: 10.1007/s10804-009-9074-y

Dyer, S., Mokoena, N., Maritz, J., \& van der Spuy, Z. (2008). Motives for parenthood among couples attending a level 3 infertility clinic in the public health sector in South Africa. Human Reproduction, 23(2), 352-357. doi: 10.1093/humrep/dem279

Finocchario-Kesslera, S., Sweata, M., Dariotisb, J., Andersonc, J., Jenningsd, J., Kellerc, J., \& Vyase, A. (2012). Childbearing motivations, pregnancy desires, and perceived partner response to a pregnancy among urban female youth: does HIV-infection status make a difference?, AIDS Care: Psychological and Socio- 
medical Aspects of AIDS/HIV, 24(1), 1-11. doi: $10.1080 / 09540121.2011 .596514$

Gerson, M. J. (1980). The lure of motherhood. Psychology of Women Quarterly, 5(2), 207-218. doi:10.1111/j.14716402.1980.tb00957.x

Gerson, M. J. (1983). A scale of motivation for parenthood: The index of parenthood motivation. The Journal of Psychology, 113(2), 211-220. doi: 10.1111/j.1471-6402.1980.tb00957.x

Gerson, M. J. (1984). Feminism and the wish for a child. Sex Role, $11(5)$ 389-399.

Recuperado

https: / / link. springer.com/article/ 10.1007\% 2FBF00287467

Ghazanfarpour, M., Arghavani, E., Khadivzade, T., Saeidi, M., Kareshki, H., Irani, M., Heidari, E., \& Dizavandi, F. (2018). Childbearing motivation in iranian engaged couples. International Journal of Pediatrics, 6(4), 7563-7568. doi: 10.22038/IJP.2018.27375.2359

Goldberg, A., Downing, J., \& Moyer, A. (2012). Why parenthood, and why now? Gay men's motivations for pursuing parenthood. Family Relations, 61(1), 157-174. doi: 10.2307/41403646

Gonçalves, S. (2016). Vinculação em jovens adultos e motivação para a parentalidade (Tesis de maestría). Universidade Portucalense Infante D. Henrique, Porto, Portugal.

Guedes, M., Pereira, M., Pires, R., Carvalho, P., \& Canavarro, M. C. (2013). Childbearing motivations scale: Construction of a new measure and its preliminary psychometric properties, Journal of Child and Family Studies, 24(1), 180-194. doi: 10.1007/s10826-013-9824-0

Hoppe, A., Fritsche, I., \& Koranyi, N. (2016). Self-transcendence as a psychological parenthood motive: When mortality salience increases the desire for non-biological children. European Journal of Social Psychology, 47(4), 1-13. doi: 10.1002/ ejsp. 2218

J ungert, T., Landry, R., Joussemet, M., Mageau, G., Gingras, I., \& Koestner, R. (2014). Autonomous and controlled motivation for parenting: associations with parent and child outcomes. Journal of Child and Family Studies, 24(7), 1932-1942. doi: 10.1007/s10826-014-9993-5

Kamalski, J., Huggett, S., Kalinaki, E., Lan, G., Lau, G., Pan, L., \& Scheerooren, S. (2015). World of research - Revealing patterns and archetypes in scientific research. Recuperado de https: // bluesyemre.files.wordpress.com/2017/03/elswor2015.pdf

Leeners, B., Neumaier-Wagner, P., Kuse, S., Merki, S., Stiller, R., Neises, M., Inthurn, B., \& Rath, W. (2009). Motivation for motherhood in women with hypertensive diseases. Journal of 
Psychosomatic Obstetrics y Gynecology, 30(2), 133-140. doi: $10.1080 / 01674820802545438$

Liefbroer, A. C. (2005). The impact of perceived costs and rewards of childbearing on entry into parenthood. European Journal of Population, 21(4), 367-391. doi: 10.1007/s10680-005-2610-y

Matias, M., \& Fontaine, A. M. (2013). Desenvolvimento e validação factorial da escala de Motivos face à parentalidade, Paidéia, 23(54), 9-20. doi: 10.1590/1982-43272354201303

Miller, W. B. (1992). Personality traits and developmental experiences as antecedents of childbearing motivation. Demography, 29(2), 265-285. doi: 10.2307/2061731

Miller, W. B. (1994). The relationship between childbearing motivations and attitude toward abortion. Family Planning Perspectives, 26(4), 165-168. doi: 10.2307/2136241

Miller, W. B. (1995). Childbearing motivations and its measurement. Journal of Biosocial Science, 27(4), 473-487. doi: $10.1017 /$ S0021932000023087

Miller, W. B., Millstein, S. G., \& Pasta, D. J. (2008). The measurement of childbearing motivation in couples considering the use of assisted reproductive technology. Biodemography and Social Biology, 54(1), 8-32. doi: 10.1080/19485565.2008.9989129

Miller, W. B., \& Pasta, D. J. (1993). Motivational and nonmotivational determinants of child-number desires, Population and environment. A J ournal of Interdisciplinary Studies, 15(2), 113136. Recuperado de http://www.jstor.org/stable/27503330

Miller, W. B., \& Rodgers, J. L. (2001). Human Bonding Systems: Their Evolutionary Origins, Neural Bases, and Psychological Manifestations. Boston, EUA: Kluwer.

Miller, W. B., Severy, L., \& Pasta, D. (2004). A framework for modelling fertility motivation in couples. A Journal of Demography, 58(2), 193-205. Recuperado de http://www.jstor.org/stable/4148229

Miller, W. Trent. M., \& Chung, S. E. (2014). Ambivalent childbearing motivations: predicting condom use by urban, AfricanAmerican, female youth. Journal of Pediatric and Adolescent Gynecology, 27(3), 151-60. doi:10.1016/j.jpag.2013.09.009

Ngu, M., Hay, M., \& Menahem, S. (2015). Why babies - What Australian mothers say. Journal of Obstetrics and Gynaecology, 35(6), 591-594. doi: 10.3109/01443615.2014.991282

Pezeshki, M. Z., Zeighami, B., \& Miller, W. B. (2005). Measuring the childbearing motivation of couples peferred to the Shiraz health center for premarital examinations. Journal of Biosocial Science, 37(1), 37-53. Recuperado de https://www.ncbi. nlm.nih.gov/pubmed/15688570 
Rabin, A. I. (1965). Motivation for parenthood. Journal of Projective Techniques and Personality Assessment, 29(4), 405-413. doi: 10.1080/0091651X.1965.10120230

Ribeiro, C. R., Gomes, R., \& Nunes, M. C. (2015). A paternidade e a parentalidade como questões de saúde frente aos rearranjos de gênero. Temaslibres, 20(11), 3589-3598. doi:10.1590/1413812320152011.19252014

Stanford, J., \& Porucznik, C. (2017). Enrollment, childbearing motivations, and intentions of couples in the creighton model effectiveness, intentions, and behaviors assessment (CEIBA) study. Frontiers in Medicine, 4(147), 1-10. doi: 10.3389/fmed.2017.00147

Stöbel-Richter, Y., Beutel, M. E., Finck, C., \& Brähler, E. (2005). The "wish to have a child", childlessness and infertility in Germany. Human Reproduction, 20(10), 2850-2857. doi: 10.1093/humrep/dei121

Todorov, J. C., \& Borges, M. M. (2005). O conceito de motivação na psicologia. Revista Brasileira de Terapia Comportamental e Cognitiva, 7(1), 119-132. doi: 10.31505/rbtcc.v7i1.47

Van Balen, F. (2004). Late parenthood among subfertile and fertile couples: motivations and educational goals. Patient Education and Counseling, 59(3), 276-282. doi: 10.1016/j.pec. 2004.09.002

Wesley, Y. (2003). Desire for children among black women with and without HIV infection. Journal of Nursing Scholarship, 35(1), 37-43. doi: 10.1111/j.1547-5069.2003.00037.x

\section{Endereço para correspondência \\ Giuliana Vásquez Varas}

Rua Praia de Botafogo, 316 apto 538, Botafogo, CEP 22250-040, Rio de Janeiro RJ, Brasil

Endereço eletrônico: giuli_vv700@hotmail.com

J uliane Callegaro Borsa

Pontifícia Universidade Católica do Rio de Janeiro

Programa de Pos-Graduación en Psicología

Rua Marquês de São Vicente, 225, Edifício Cardeal Leme, 2o andar, Gávea, CEP 22451-900, Rio de Janeiro - RJ, Brasil

Endereço eletrônico: juliborsa@gmail.com

Recebido em: 18/12/2018

Reformulado em: 28/03/2019

Aceito em: $28 / 03 / 2019$

\section{Notas}

* Psicóloga, Master en Psicología Clínica de la Universidad de San Martin de Porres y doctoranda en Psicología Clínica de la Pontificia Universidad Católica do Rio de Janeiro (PUC-Rio). 
** Profesora Adjunta II del Departamento de Psicologia del Programa de PosGraduación en Psicología Clínica de la Pontificia Universidad Católica de Rio de J aneiro (PUC-Rio), Master en Psicología Clínica por la Pontificia Universidad Católica de Rio Grande do Sul (PUCRS), Doctorado y Pos-Doctorado en Psicología por la Universidad Federal de Rio Grande do Sul (UFRGS).

Este artigo de revista Estudos e Pesquisas em Psicologia é licenciado sob uma Licença Creative Commons Atribuição-Não Comercial 3.0 Não Adaptada. 